Research Paper

\title{
Zwitterionic Poly(carboxybetaine)-based Cationic Liposomes for Effective Delivery of Small Interfering RNA Therapeutics without Accelerated Blood Clearance Phenomenon
}

\author{
Yan Li 1,2, ${ }^{*}$, Ruiyuan Liu 1,3, ${ }^{*}$, Yuanjie Shi ${ }^{4}$, Zhenzhong Zhang $3{ }^{3, 凶}$, Xin Zhang $1, \bowtie$ \\ 1. National Key Laboratory of Biochemical Engineering, Institute of Process Engineering, Chinese Academy of Sciences, Beijing, 100190, \\ China \\ 2. University of Chinese Academy of Sciences, Beijing, 100049, China \\ 3. School of Pharmaceutical Sciences, Zhengzhou University, Zhengzhou, 450001, China \\ 4. School of Life Sciences, University of Beijing Institute of Technology, Beijing, 100081, China
}

* These authors contributed equally to this work.

$\square$ Corresponding authors: zhangzz08@126.com and xzhang@ipe.ac.cn

() 2015 Ivyspring International Publisher. Reproduction is permitted for personal, noncommercial use, provided that the article is in whole, unmodified, and properly cited. See http://ivyspring.com/terms for terms and conditions.

Received: 2014.12.03; Accepted: 2015.01.23; Published: 2015.02.27

\begin{abstract}
For efficient delivery of small interfering RNA (siRNA) to the target diseased site in vivo, it is important to design suitable vehicles to control the blood circulation of siRNA. It has been shown that surface modification of cationic liposome/siRNA complexes (lipoplexes) with polyethylene glycol (PEG) could enhance the circulation time of lipoplexes. However, the first injection of PEGylated lipoplexes in vivo induces accelerated blood clearance and enhances hepatic accumulation of the following injected PEGylated lipoplexes, which is known as the accelerated blood clearance $(A B C)$ phenomenon. Herein, we developed zwitterionic poly(carboxybetaine) (PCB) modified lipoplexes for the delivery of siRNA therapeutics, which could avoid protein adsorption and enhance the stability of lipoplexes as that for PEG. Quite different from the PEGylation, the PCBylated lipoplexes could avoid ABC phenomenon, which extended the blood circulation time and enhanced the tumor accumulation of lipoplexes in vivo. After accumulation in tumor site, the PCBylation could promote the cellular uptake and endosomal/lysosomal escape of lipoplexes due to its unique chemical structure and $\mathrm{pH}$-sensitive ability. With excellent tumor accumulation, cellular uptake and endosomal/lysosomal escape abilities, the PCBylated lipoplexes significantly inhibited tumor growth and induced tumor cell apoptosis.
\end{abstract}

Key words: Cationic liposomes, siRNA therapeutics, Poly(carboxybetaine), Polyethylene glycol, Accelerated blood clearance (ABC) phenomenon.

\section{Introduction}

Despite small interfering RNA (siRNA) has garnered much interest as a potential drug for cancer therapy, the clinical application of siRNA still faces considerable obstacles due to its inherent problems such as instability in physiological environment, poor membrane permeability and short serum half-life [1-4]. Therefore, toward the success in siRNA therapeutics, it is significant to develop efficient and safe vectors to extend the circulation time of siRNA and deliver siRNA into cytoplasm of the target cells $[5,6]$. 
Among them, cationic liposomes have been reported to display a strong ability to protect siRNA from RNase degradation and to accumulate in tumor via the enhanced permeability and retention (EPR) effects [7-9]. However, cationic liposome/siRNA complexes (lipoplexes) are prone to have nonspecific interaction with plasma protein in vivo and are rapidly cleared by the reticuloendothelial system (RES) before reaching the tumor site, resulting in low gene silencing efficiency $[10,11]$.

One strategy to circumvent this problem is the use of polyethylene glycol (PEG)-lipids to shield the positive charge on the lipoplexes surface to increase the serum stability and blood circulation time of lipoplexes [12-14]. Unfortunately, the steric hindrance due to PEGylation reduces the electrostatic interactions required for siRNA encapsulation, cellular uptake and endosomal/lysosomal escape of the lipoplexes [15]. Most importantly, it has been shown that upon subsequent administrations of PEGylated liposomes, an immune response could be elicited, leading to a rapid blood clearance, which further compromised the lipoplexes in clinical application [16]. PEGylated lipoplexes are intended to stimulate the spleen to produce anti-PEG IgM after the first administration, which selectively binds to PEG on the surface of the second administrated lipoplexes to cause rapid blood elimination and enhanced hepatic uptake (Scheme 1) [17-19]. This effect is known as the "accelerated blood clearance (ABC) phenomenon" [20-22]. The ABC phenomenon represents a tremendous challenge to the clinical application of PEGylated lipoplexes due to the accelerated blood clearance and decreased target diseased site accumulation [23].

There have been many strategies to avoid the induction of $\mathrm{ABC}$ phenomenon through changing the physicochemical properties of the PEGylated lipo- somes. Semple and co-workers used PEG-lipids (PEG-CerC14) with a smaller C14 lipid anchor to modify liposomes to abrogate $\mathrm{ABC}$ phenomenon, which could exchange out of the carrier immediately after injection, with greater than 50\% loss of PEG-lipid in approximately $3 \mathrm{~min}$. However, the lipid exchange would result in the defects in the membranes of the liposomes [24]. It has also been shown that liposomes modified with cleavable PEG-lipid derivatives (PEG-CHEMS and PEG-CHMC) could lessen or eliminate the $\mathrm{ABC}$ phenomenon. The cleavable PEG-lipid derivatives were constructed by linking PEG to lipids through ester bonds, and the chemical bond would gradually be cleaved during circulation. However, the effect on long circulation of the cleavable PEGylated liposomes was worse than that of non-cleavable PEGylated liposomes [25].

Therefore, it is urgently needed to develop a strategy to avoid the immunogenicity of PEGylation without significantly limiting the in vivo performance of liposomes. Zwitterionic polymers such as poly (sulfobetaine) (PSB) and poly (carboxybetaine) (PCB) have been demonstrated to be promising alternatives to PEG due to their ability to resist nonspecific protein adsorption [26, 27]. In our previous study, it has been shown that zwitterionic polymer PCB based lipid DSPE-PCB 20 had the same ability to enhance the serum stability of lipoplexes. However, quite different from the PEGylation, zwitterionic DSPE- PCB $_{20}$ could offer stability without interfering with the siRNA encapsulation efficiency and endosomal/lysosomal escape ability of lipoplexes, which was favorable for the systemic delivery of siRNA [28]. However, whether PCBylation could avoid ABC phenomenon and enhance the target tumor accumulation of lipoplexes in vivo has not been verified.

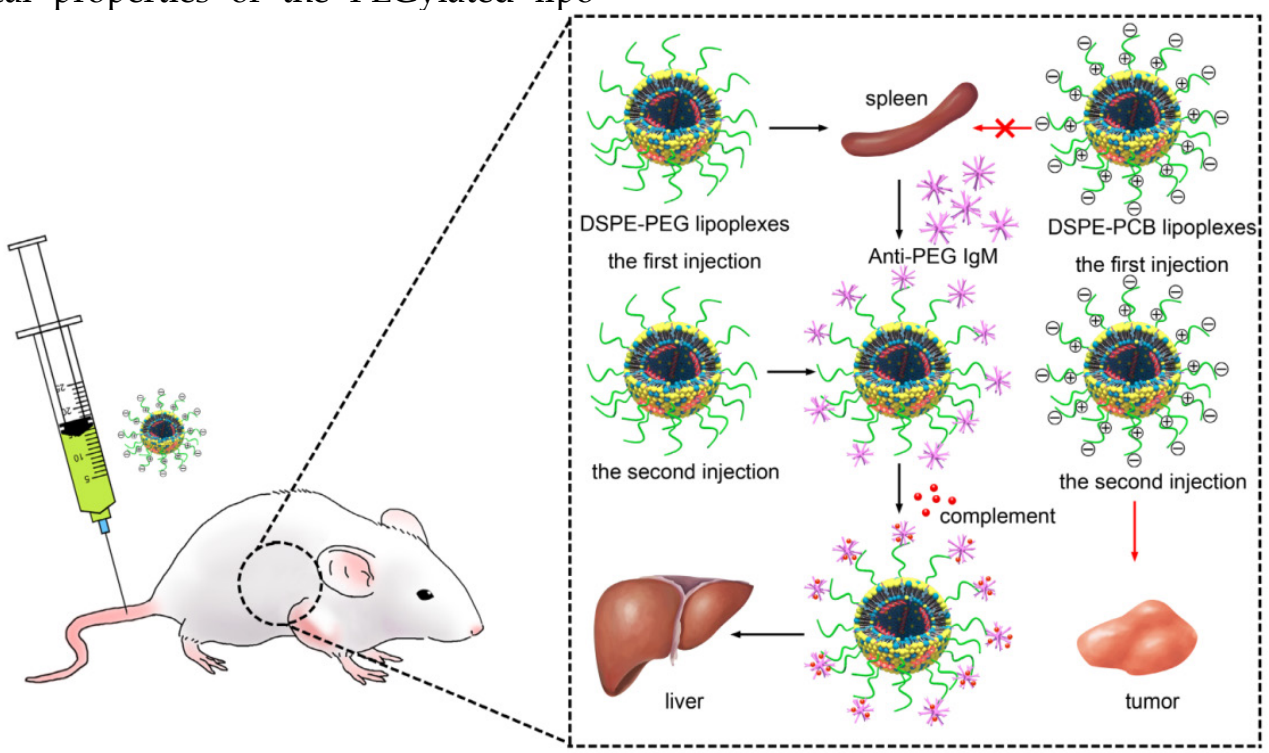

Scheme 1. Schematic illustration of the ABC process of the PEGylated and PCBylated lipoplexes. 
In this study, cationic liposomes based siRNA delivery system with the modification of PCB was developed. The performances of PCBylated lipoplexes in pharmacokinetics, biodistribution and tumor therapy were investigated. Our findings demonstrated that PCBylation could avoid ABC phenomenon, which facilitated the tumor accumulation and therefore enhanced the antitumor activity of lipoplexes in vivo (Scheme 1).

\section{Materials and Methods}

\section{Materials}

Dimethyldioctadecylammonium

bromide

(DDAB), cholesterol (95\%) and 4', 6-diamidino-2-phenylindole dihydrochloride (DAPI) were purchased from J\&K Scientific Ltd. 1, 2-Distearoyl-sn-glycerol-3-phosphoethanolamine-N-[ methoxy (polyethylene glycol)-2000] (DSPE-PEG 2000) was from Shanghai Advanced Vehicle Technology Ltd. Co. LysoTracker Red was obtained from Invitrogen. Glo lysis buffer $(\times 1)$ was purchased from Promega. Fluorescence labeled Cy5-labeled siRNA (antisense strand, 5' -UUUGAAGUAUGCCUCAAGG dTdT-3'), FAM-labeled siRNA (antisense strand, 5'-UUUGAAGUAUGCCUCAAGGdTdT-3'), as well as siRNA targeting Plk1 mRNA (siPlk1, antisense strand, 5'-UAAGGAGGGUGAUCUUCUUCAdTdT3') were synthesized by Suzhou Ribo Life Science Co. (Kunshan, China). All other reagents used were obtained commercially at analytical grade.

\section{Animals}

Male SD rats weighting 150-200 $\mathrm{g}$ and female $\mathrm{BALB} / \mathrm{c} \mathrm{nu/nu}$ mice weighting 20-25 g were purchased from the Academy of Military Medical Sciences of China. The animal had free access to water and animal chow. All care and handing of animals was performed in accordance with the protocols approved by the Institutional Animals Care and Use Committee of Peking University.

\section{Preparation and characterization of cationic liposomes and lipoplexes}

Distearoyl phosphoethanolamine-poly(carboxybetaine) ${ }_{20}$ (DSPE-PCB 20 ) was used to make modification of cationic liposomes as it had comparable ability in enhancing the serum stability of lipoplexes with that of DSPE-PEG 2000 in our previous work [28]. Cationic liposomes with composition of $\mathrm{DDAB} /$ cholesterol/DSPE-PCB 20 or $\mathrm{DDAB} /$ cholesterol/DSPE-PEG 2000 (1/1/0.2, molar ratio) were prepared by thin lipid film method. Briefly, all lipids were dissolved in chloroform and were subsequently evaporated to form dry film using a rotary evaporator under vacuum at $45{ }^{\circ} \mathrm{C}$. The resulting thin lipid film was then hydrated with phosphate buffered saline (PBS, $\mathrm{pH}=7.4$ ) and sonicated at $37{ }^{\circ} \mathrm{C}$ for $30 \mathrm{~min}$. Finally, the cationic liposomes were sized by repeated extrusion through EmulsiFlex-C5 high-pressure homogenizer (Avestin, Canada). The lipoplexes were prepared by mixing cationic liposomes with siRNA together at designed $\mathrm{N} / \mathrm{P}$ ratios at room temperature for $30 \mathrm{~min}$. The mean diameter and zeta potential of the resulting cationic liposomes and lipoplexes in DMEM were determined using a Malvern Nano ZS instrument (Malvern Instruments). The morphological analysis was carried out by cryogenic transmission electron microscopy (Cryo-TEM, FEI Tecnai 20, The Netherlands).

\section{siRNA encapsulation efficiency}

The encapsulation efficiency of siRNA in the lipoplexes was evaluated by Quant-iT ${ }^{\mathrm{TM}}$ RiboGreen $^{\circledR}$ RNA Reagent (Invitrogen). The amount of un-encapsulated siRNA in supernatant was measured after centrifugation at $5000 \times \mathrm{g}$ for $5 \mathrm{~min}$. For low-range assay, $100 \mu \mathrm{L}$ of the reagent was added to mircoplate wells and the samples were quantified using microplate reader (SpectraMax M5, Molecular Devices, CA, USA) with the excitation wavelength of $485 \mathrm{~nm}$ and emission wavelength of $530 \mathrm{~nm}$. The encapsulation efficiency of siRNA was calculated using the formula:

Encapsulation efficiency $(\%)=\left(\mathrm{F}_{0}-\mathrm{F}_{1}\right) / \mathrm{F}_{0} \times 100 \%$

Where $\mathrm{F}_{0}$ was the fluorescence of siRNA used for encapsulation, $F_{1}$ was the fluorescence of siRNA in supernatant.

\section{Serum stability of lipoplexes}

The serum stability of lipoplexes was examined by incubating lipoplexes in DMEM containing 10\% FBS at $37^{\circ} \mathrm{C}$ under gentle stirring. At predetermined time points, the mean diameter of the lipoplexes was monitored by DLS.

\section{The protonation of DSPE-PCB ${ }_{20}$ lipoplexes in acidic environment}

The ability of protonation in acidic environment for DSPE-PCB 20 lipoplexes was determined by incubating lipoplexes in PBs solution with different $\mathrm{pH}$ from 7.4 to 3.5 at $37^{\circ} \mathrm{C}$ for $30 \mathrm{~min}$. The zeta potential of the lipoplexes was assessed with a Zetasizer Nano ZS instrument.

\section{Cytotoxicity of lipoplexes}

The cytotoxicity of lipoplexes was assessed with a MTT viability assay against Hela cells. Hela cells were seeded at $1 \times 10^{4}$ cells per well on a 96-well plate and allowed to adhere overnight at $37^{\circ} \mathrm{C}$ for $24 \mathrm{~h}$. Afterwards, $100 \mu \mathrm{L}$ of various concentration of DSPE-PEG and DSPE-PCB ${ }_{20}$ lipoplexes at N/P ratio of 
5/1 with DMEM containing 10\% FBS were added to each well and incubated with the cells for 24 h. $20 \mu \mathrm{L}$ of MTT stock solution $(5 \mathrm{mg} / \mathrm{mL})$ was then added to each well and incubated for an additional $2 \mathrm{~h}$. After incubation, the medium was replaced with $100 \mu \mathrm{L}$ of DMSO and the absorbance was read at $490 \mathrm{~nm}$ using a microplate reader (Tecan, Switzerland). Cell viability was normalized to that of Hela cells cultured in the culture medium with PBS treatment.

\section{Flow cytometry measurement}

The cellular uptake of the lipoplexes was evaluated by flow cytometry. Briefly, Hela cells were seeded in 12-well plates at $1 \times 10^{5}$ cells per well in 500 $\mu \mathrm{L}$ of culture medium and allowed to adhere for $24 \mathrm{~h}$. FAM-labeled siRNA was complexed with cationic liposomes at $\mathrm{N} / \mathrm{P}$ ratio of $5 / 1$ for $30 \mathrm{~min}$ at room temperature and then added to each well with the DMEM containing $10 \%$ FBS with the final concentration of $2 \mu \mathrm{g}$ siRNA/mL. The lipoplexes were incubated with cells for $0.25,0.5,1,1.5$ and $2.5 \mathrm{~h}$, respectively. Afterwards, the medium was removed and cells were washed three times with cold PBS, trypsinezed and harvested in PBS for flow cytometry analysis using BD Calibur flow cytometry (BD Co., USA).

To further elucidate the mechanisms underlying the cellular internalization of DSPE-PEG and DSPE-PCB 20 lipoplexes, the cellular uptake study was performed at $4{ }^{\circ} \mathrm{C}$ or in the presence of various endocytic inhibitors. Briefly, Hela cells were pre-incubated with the endocytic inhibitors for 30 min, and the lipoplexes with FAM-labeled siRNA at $\mathrm{N} / \mathrm{P}$ ratio of $5 / 1$ were added for $2 \mathrm{~h}$ uptake experiment at $37^{\circ} \mathrm{C}$. Afterwards, the medium was removed and cells were washed three times with cold PBS, trypsinezed and harvested in PBS for flow cytometry analysis using BD Calibur flow cytometry (BD Co., USA). Results were expressed as percentage uptake level of control cells that were incubated with lipoplexes at $37^{\circ} \mathrm{C}$ for $2 \mathrm{~h}$ in the absence of endocytic inhibitors.

\section{Confocal microscopy}

Cellular internalization and location of cationic liposomes with FAM-labeled siRNA were tested using confocal microscopy. Briefly, Hela cells were seeded in culture dish and incubated for $24 \mathrm{~h}$. Afterwards, DSPE-PEG and DSPE-PCB 20 lipoplexes at N/P ratio of 5/1 including $1 \mu \mathrm{g}$ FAM-labeled siRNA were added to each well and incubated with the cells for $2 \mathrm{~h}$ and $8 \mathrm{~h}$, respectively. Subsequently, the cells were washed three times with PBS followed by staining with LysoTracker Red for $30 \mathrm{~min}$ at $37^{\circ} \mathrm{C}$. After another three rinses with PBS, the cells were fixed by $4 \%$ paraformaldehyde for $10 \mathrm{~min}$ and the nuclei were stained using DAPI for $10 \mathrm{~min}$. The cells were then detected using a Zeiss LSM780 confocal microscopy (Zeiss Co., Germany).

\section{Quantitative evaluation of intracellular siRNA release}

To determine the amount of siRNA release from DSPE-PEG and DSPE-PCB ${ }_{20}$ lipoplexes inside the cells, Hela cells were seeded in 12-well plates $\left(1 \times 10^{5}\right)$ for $24 \mathrm{~h}$. The cationic liposome/FAM-labeled siRNA complexes were added to each well with the concentration of $2 \mu \mathrm{g} / \mathrm{mL}$ and then incubated for $8 \mathrm{~h}$. At designed time point, the cells were washed with PBS twice and lysed in $100 \mu \mathrm{L}$ of lysis buffer for $30 \mathrm{~min}$. After the centrifugation of lysates at $15000 \times \mathrm{g}$ for 15 min at $4{ }^{\circ} \mathrm{C}, 50 \mu \mathrm{L}$ of the supernatant was transferred to a black 96-well plate for fluorescence measurement using microplate reader with the excitation wavelength of $488 \mathrm{~nm}$ and emission wavelength of $525 \mathrm{~nm}$. The percentage of released siRNA of all internalized siRNA was calculated using the following equation:

$$
\text { siRNA released }(\%)=\left(\mathrm{F}_{1}-\mathrm{F}_{2}\right) /\left(\mathrm{F}_{0}-\mathrm{F}_{2}\right) \times 100 \%
$$

Where $F_{0}$ was the fluorescence of all internalized siRNA (lysis buffer is Glo lysis buffer and 2\% SDS), $\mathrm{F}_{1}$ was the fluorescence of siRNA in supernatant (lysis buffer is only Glo lysis buffer), $\mathrm{F}_{2}$ was the fluorescence induced by untreated cells.

\section{Blood clearance and tissue accumulation of lipoplexes}

To determine the blood clearance and tissue accumulation of lipoplexes, SD rats were randomly divided into 5 groups $(n=3)$. For the first injection, rats were intravenously injected with DSPE-PEG and DSPE-PCB 20 lipoplexes at a dose of $1 \mathrm{mg}$ Cy5-labeled siRNA/ kg. Control animals received PBS instead of lipoplexes. Five days later, the same lipoplexes were injected intravenously to the same rats at a dose of 1 mg Cy5-labeled siRNA $/ \mathrm{kg}$. At selected post-injection time points after two injections, blood $(200 \mu \mathrm{L})$ was sampled via eye puncture. The liver and spleen were removed $1 \mathrm{~h}$ after the injection. Plasma was collected after centrifugation at $903 \times \mathrm{g}$ for $10 \mathrm{~min}$ at $4{ }^{\circ} \mathrm{C}$. The concentration of the Cy5-labeled siRNA in plasma was detected using microplate reader (SpectraMax M5, Molecular Devices, CA, USA) for fluorescence measurement after demulsification with $2 \%$ Tween 80. The distribution of Cy5-labeled siRNA in liver and spleen was detected using a Kodak in vivo imaging system (Kodak In-Vivo Imaging System FX Pro. Carestream Health, USA).

\section{Detection of IgM and IgG protein level}

SD Rats were pre-treated with DSPE-PEG and 
DSPE-PCB 20 lipoplexes $(\mathrm{N} / \mathrm{P}=5 / 1)$ at a dose of $1 \mathrm{mg}$ Cy5-labeled siRNA/kg ( $=5)$. Five days later, blood samples were collected via eye puncture. Plasma was collected after centrifugation at $903 \times \mathrm{g}$ for $10 \mathrm{~min}$ at 4 ${ }^{\circ} \mathrm{C}$. The IgM level in plasma was determined using ELISA method according to the manufacturer's instructions (Rat IgM Ready-SET-Go! ®, eBioscience). The absorbance was measured at $450 \mathrm{~nm}$ using a microplate reader (SpectraMax M5, Molecular Devices, CA, USA).

$200 \mu \mathrm{L}$ of plasma samples collected on day 5 after the first injection of DSPE-PEG lipoplexes were incubated with $50 \mu \mathrm{L}$ of PBS, DSPE-PEG and DSPE-PCB 20 lipoplexes at $37^{\circ} \mathrm{C}$ for $15 \mathrm{~min}$. After incubation, lipoplexes were separated out by centrifugation at $14462 \times \mathrm{g}$ for $30 \mathrm{~min}$. The IgM in supernatant was detected using ELISA method according to the manufacturer's instructions (Rat IgM Ready-SET-Go! $®$, eBioscience). The absorbance was measured at 450 $\mathrm{nm}$ using a microplate reader.

To detect the IgG protein level, other SD rats were pre-treated with two injections of DSPE-PEG and DSPE-PCB 20 lipoplexes $(\mathrm{N} / \mathrm{P}=5 / 1)$ at a dose of 1 mg Cy5-labeled siRNA/kg $(n=5)$, respectively. The time interval between the two injections was 7 days. Two weeks after the second injection, blood samples were collected via eye puncture. Plasma was collected after centrifugation at $903 \times \mathrm{g}$ for $10 \mathrm{~min}$ at $4{ }^{\circ} \mathrm{C}$. The IgG level in plasma was determined using ELISA method (Rat IgG total Ready-SET-Go! ® according to the manufacturer's instruction. The absorbance was measured at $450 \mathrm{~nm}$ using a microplate reader (SpectraMax M5, Molecular Devices, CA, USA).

The standard curves of IgM and IgG level were shown in Supplementary Material: Figure S1.

\section{Biodistribution of lipoplexes in tumor-bearing nude mice}

To detected the biodistribution of DSPE-PEG and DSPE-PCB $\mathrm{PB}_{20}$ lipoplexes in Hela tumor-bearing nude mice, mice were randomly divided into 4 groups $(n=10)$. For the first injection, mice were intravenously injected with PBS, Cy5-labeled siRNA, DSPE-PEG and DSPE-PCB $20 / C y 5$-labeled siRNA lipoplexes $(\mathrm{N} / \mathrm{P}=5 / 1)$ at a dose of $0.5 \mathrm{mg}$ Cy5-labeled siR$\mathrm{NA} / \mathrm{kg}$, respectively. $24 \mathrm{~h}$ after the first injection, five mice of each group were sacrified and the main organs and tumor were harvested. Five days later, the same samples were injected intravenously to the same mice at a dose of $0.5 \mathrm{mg}$ Cy5-labeled siRNA $/ \mathrm{kg}$. At 24 $\mathrm{h}$ post-injection, the mice were sacrificed and the main organs and tumor were harvested. The biodistribution of Cy5-labeled siRNA in organs and tumor was detected using a Kodak in vivo imaging system (Ko- dak In-Vivo Imaging System FX Pro. Carestream Health, USA).

To further quantify the accumulated dose of Cy5-labeled siRNA in liver and tumor, the excised tissues were homogenized in 300 or $1000 \mu \mathrm{L}$ lysis buffer $(300 \mu \mathrm{L}$ for tumors and $1000 \mu \mathrm{L}$ for livers) and incubated at $65{ }^{\circ} \mathrm{C}$ for $10 \mathrm{~min} .100 \mu \mathrm{L}$ of supernatant was collected after centrifugation at $16162 \times \mathrm{g}$ for 10 min. Cy5-labeled siRNA in supernatant was extracted and quantified by microplate reader (SpectraMax M5, Molecular Devices, CA, USA) with the excitation wavelength of $649 \mathrm{~nm}$ and emission wavelength of $670 \mathrm{~nm}$. The accumulated dose in each tissue was calculated from a standard curve obtained by extracting known amounts of Cy5-labeled siRNA in tissues obtained from un-injected animals [29].

\section{Tumor suppression study}

The anti-tumor efficacy was investigated using Hela tumor-bearing nude mice. When the tumor volume reached approximately $50 \mathrm{~mm}^{3}$, mice were randomly divided into four groups $(\mathrm{n}=5)$ and treated with PBS, siPlk1, DSPE-PEG and DSPE-PCB 20 lipoplexes with siPlk1 at a dose of $2.5 \mathrm{mg} / \mathrm{kg}$ every other day, respectively. Body weight and tumor volume were monitored before each injection. The tumor volume was measured using the formula:

$$
\mathrm{V}\left(\mathrm{mm}^{3}\right)=\left(\mathrm{a} \times \mathrm{b}^{2}\right) / 2
$$

Where $\mathrm{a}$ and $\mathrm{b}$ are the major and minor axes of the tumor, respectively.

At $24 \mathrm{~h}$ post-injection, the mice were sacrificed and the main organs and tumor were harvested. The level of Plk1 mRNA and protein in tumor was analyzed by qRT-PCR and WB. The apoptosis of tumor sections was observed after staining with terminal deoxynucleotidyl transferase-mediated dUTP nick end labeling (TUNEL) assay.

\section{Statistical analysis}

Quantitative data were expressed as mean \pm SD. Means were compared using Student's $t$ test. $p$ values $<0.05$ were considered statistically significant.

\section{Results and Discussion}

\section{The characteristics of cationic liposomes}

Zwitterionic polymer based lipid distearoyl phosphoethanolamine-poly (carboxybetaine) ${ }_{20}$ (DSPE-PCB ${ }_{20}$ ) was used to make modification of cationic liposomes as it had comparable ability in enhancing the serum stability of cationic liposomes with that of DSPE-PEG 2000 in our previous work (Figure 1A) [28]. As shown in Figure 1B, DSPE-PEG and DSPE-PCB ${ }_{20}$ cationic liposomes had similar diameters with narrow distributions, about $119.2 \pm 16.2 \mathrm{~nm}$ and 
$113.2 \pm 20.9 \mathrm{~nm}$, respectively. The morphology of cationic liposomes was further observed by Cryo-TEM. The Cryo-TEM images in Figure 1C showed that both DSPE-PEG and DSPE-PCB ${ }_{20}$ cationic liposomes had spherical shape with an average size of $80 \mathrm{~nm}$, which is slightly smaller than that measured by DLS due to the dehydration during sample preparation. The surface zeta potentials of both PEGylated and PCBylated cationic liposomes in DMEM medium were comparable, about $10.2 \pm 2.7 \mathrm{mV}$ and $14.2 \pm 3.0 \mathrm{mV}$, which is significantly lower than that of unmodified cationic liposomes $(33.5 \pm 4.5 \mathrm{mV})$ with the same molar ratio of DDAB and cholesterol (Figure 1B). The results indicated that PCB had the same ability to reduce the surface charge of cationic liposomes as PEG did.

A

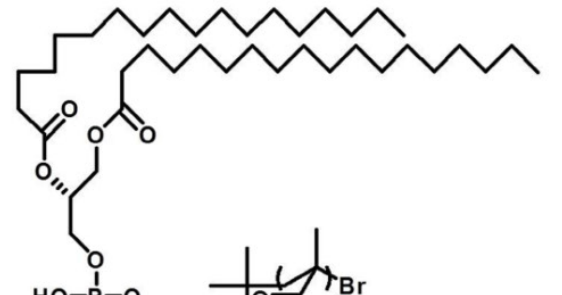

The positive charge of cationic liposomes allowed for binding of negatively charged siRNA to form lipoplexes. To assess the siRNA binding ability of DSPE-PEG and DSPE-PCB 20 cationic liposomes, percentage of siRNA encapsulation was further determined by Quant-iT ${ }^{\mathrm{TM}}$ RiboGreen ${ }^{\circledR}$ RNA Reagent. The encapsulation efficiency was only $14.6 \pm 5.4 \%$ for DSPE-PEG cationic liposomes at N/P ratio of $1 / 1$, while the encapsulation efficiency could reach to $49.0 \pm 5.3 \%$ for DSPE-PCB 20 cationic liposomes (Figure 1D). The excellent siRNA binding ability of ${ }^{D S P E}-\mathrm{PCB}_{20}$ cationic liposomes might due to the unique structure of zwitterionic polymers that the cationic quaternary amine groups assisted the cationic liposomes in retaining the siRNA [30].

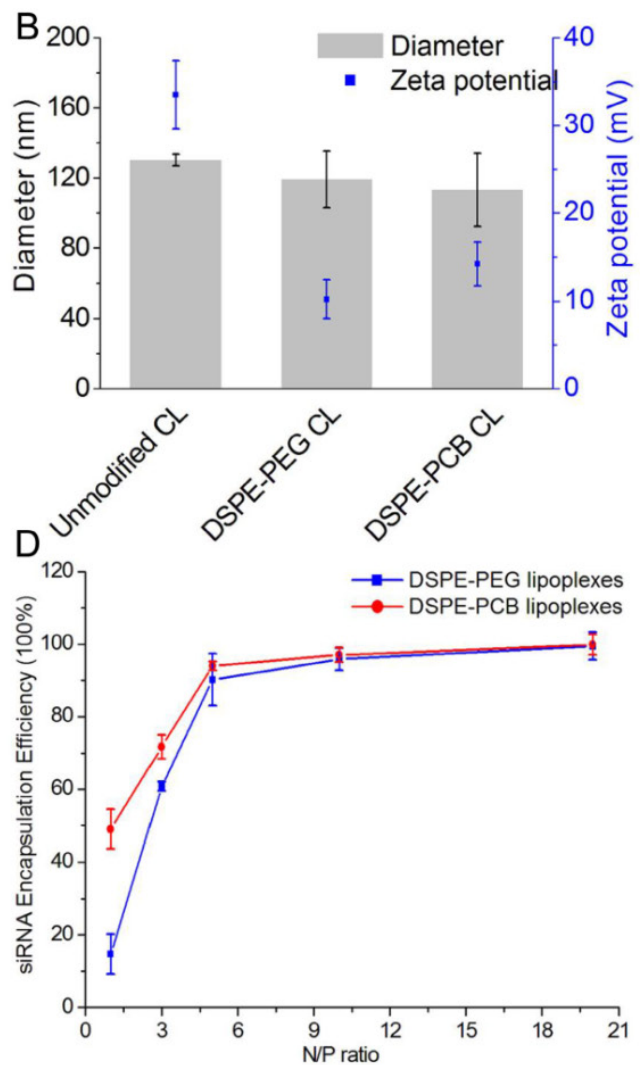

C
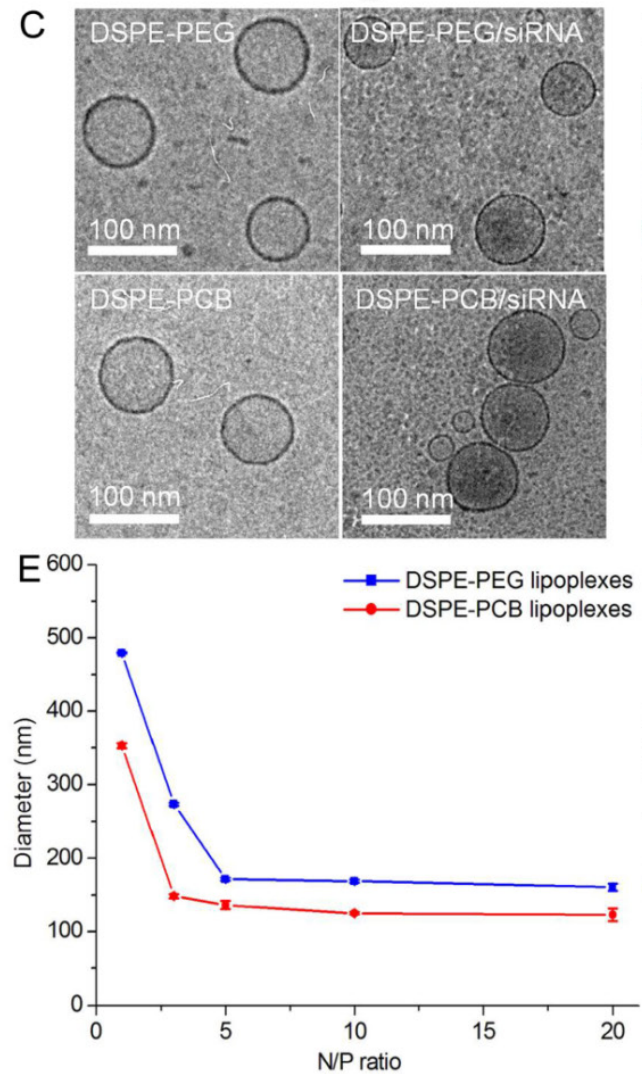

Figure 1. Characterization of cationic liposomes and lipoplexes. (A) The chemical structure of DSPE-PCB20. (B) Mean particle diameter and zeta potential of cationic liposomes in DMEM. (C) Cryo-TEM images of cationic liposomes and lipoplexes at N/P ratio of 5/1. (D) The siRNA encapsulation efficiency of lipoplexes evaluated by Quant-iTTM RiboGreen ${ }^{\circledR}$ RNA Reagent. (E) The mean diameter of lipoplexes at various N/P ratios in DMEM. (F) The zeta potential of lipoplexes at various N/P ratios in DMEM. Data are shown as the mean \pm S.D. of three independent experiments. 
The size and surface property of lipoplexes are important physiochemical parameters in designing the cancer-targeting delivery system [31]. As shown in Figure 1E, the diameter of both lipoplexes was decreased with the increase of $\mathrm{N} / \mathrm{P}$ ratio. It was about $100-200 \mathrm{~nm}$ when the $\mathrm{N} / \mathrm{P}$ ratio ranging from $3 / 1$ to $20 / 1$, which was suitable for the prolonged blood circulation to perform the EPR effect [32]. On the contrary, the corresponding zeta potential values of lipoplexes were increased with the increase of $\mathrm{N} / \mathrm{P}$ ratio. As shown in Figure 1D, the siRNA encapsulation efficiency of lipoplexes was low at N/P ratio of $1 / 1$ or $3 / 1$, which was not favorable for siRNA delivery. When the $\mathrm{N} / \mathrm{P}$ ratio reached $5 / 1$ or larger than $5 / 1$, the siRNA encapsulation efficiency was nearly $100 \%$. However, extra cationic liposomes could induce cytotoxicity $[33,34]$. Therefore, lipoplexes with $\mathrm{N} / \mathrm{P}$ ratio of $5 / 1$ were chosen for the following experiments as they had appropriate siRNA encapsulation efficiency, size and zeta potentials characteristics. Additionally, both DSPE-PEG and DSPE-PCB 20 lipoplexes at N/P ratio of 5/1 had spherical shape (Figure 1C).

\section{Serum stability of lipoplexes}

The instability of unmodified lipoplexes when exposed to plasma proteins impeded the clinical use of cationic liposomes as systemic delivery vehicles of siRNA [35]. Therefore, the serum stability of DSPE- $\mathrm{PCB}_{20}$ lipoplexes was investigated whether the PCB modification could overcome this drawback. $\mathrm{DSPE}^{\mathrm{PCB}} \mathrm{B}_{20}$ lipoplexes at $\mathrm{N} / \mathrm{P}$ ratio of $5 / 1$ were incubated with DMEM containing $10 \%$ FBS, and the changes in the sizes of the lipoplexes were analyzed by DLS measurements. As shown in Figure 2 and Supplementary Material: Figure S2, both DSPE-PCB ${ }_{20}$ and DSPE-PEG lipoplexes exhibited excellent serum stability as demonstrated by the consistent diameter after incubation for $84 \mathrm{~h}$ at $37^{\circ} \mathrm{C}$. The results indicated that the presence of PCB on the surface of lipoplexes prevented nonspecific protein adsorption and aggregation of the lipoplexes as PEG did.

\section{In vitro cytotoxicity of lipoplexes}

The biocompatibility of lipoplexes was crucial for its clinical application. Therefore, the cytotoxicity of DSPE-PEG and DSPE-PCB 20 lipoplexes to Hela cells was evaluated using the MTT assay. As shown in Figure 3, cell viability of DSPE-PEG and DSPE-PCB ${ }_{20}$ lipoplexes at $\mathrm{N} / \mathrm{P}$ ratio of $5 / 1$ remained nearly $90 \%$ when the siRNA concentration reached $8 \mu \mathrm{g} / \mathrm{mL}$. The results suggested that $\mathrm{DSPE}-\mathrm{PCB}_{20}$ lipoplexes had good biocompatibility as that for DSPE-PEG lipoplexes, which were suitable for in vivo application.

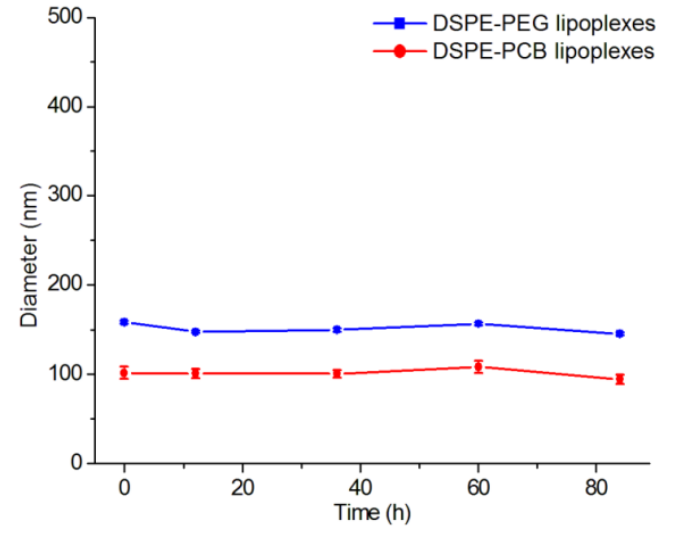

Figure 2. The changes of lipoplexes sizes in the presence of $10 \%$ FBS with the extension of time. Data are shown as the mean \pm S.D. of three independent experiments.

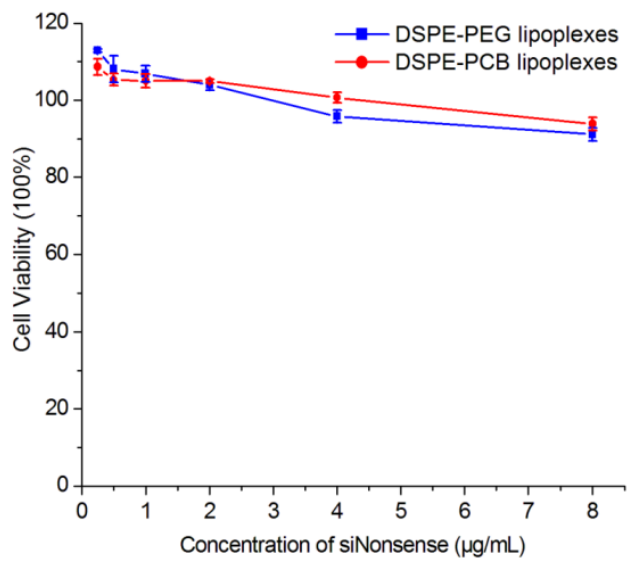

Figure 3. Cytotoxicity of DSPE-PEG and DSPE-PCB 20 cationic liposomes with siNonsense to Hela cells.

\section{ABC phenomenon of PCBylated lipoplexes}

PEGylated cationic liposome/siRNA complexes could induce $A B C$ phenomenon after repeated administration, which resulted in enhanced liver accumulation and decreased tumor accumulation of lipoplexes [36, 37]. The ABC phenomenon significantly impeded the clinical use of PEGylated lipoplexes [38]. In our previous work, it has been shown that PCBylation could overcome the barriers associated with the use of PEGylation in cellular level [28]. However, whether PCBylated lipoplexes would avoid ABC phenomenon in vivo has not been verified. To answer the question, two doses of DSPE- $\mathrm{PCB}_{20}$ lipoplexes were injected into rats with a time interval of 5 days. As shown in Figure 4A, pretreatment rats with DSPE-PEG lipoplexes triggered a rapid clearance of the second dose from the circulatory system, in which siRNA concentration in plasma dropped to $48.74 \%$ ID within 5 min. Additionally, 1 hour's biodistribution after the second injection showed a highly increased liver uptake, about 2 times of that for the first injection (Figure 4C). However, the blood clearance trend and 
hepatic uptake of DSPE-PCB ${ }_{20}$ lipoplexes were basically the same for the two injections (Figure $4 \mathrm{~B}$ and 4D). The half-life of the two injections for DSPE-PEG lipoplexes and DSPE-PCB 20 lipoplexes was calculated according the siRNA concentration profiles, respectively. The half-life of DSPE-PEG lipoplexes for the first injection was $18.72 \mathrm{~h}$, and only $6.45 \mathrm{~h}$ for the second injection, while it was about $20.05 \mathrm{~h}$ for both injections of DSPE-PCB 20 lipoplexes. The results indicated that PCBylation could avoid ABC phenomenon and enhance the blood circulation time of lipoplexes.

It has been confirmed that there was a positive correlation between the anti-PEG IgM production and the accelerated clearance of the PEGylated liposomes $[39,40]$. Whether the PCBylated lipoplexes would initiate the production of anti-PCB IgM was evaluated by administration of DSPE-PCB 20 lipoplexes on rats. The plasma IgM level was detected using ELISA 5 days later. DSPE-PEG lipoplexes obviously induced the production of IgM, which was about 4 times of that for PBS groups (Figure 5A). In contrast, the IgM level has no significant difference compared with the PBS groups for the DSPE- $\mathrm{PCB}_{20}$ lipoplexes. The results demonstrated that PCBylation would not stimulate the production of IgM, and therefore would avoid the $\mathrm{ABC}$ phenomenon against the following administration.

The IgM level reached peaked after 5 days of the first injection and it has also been shown that the IgG protein level continued to increase with additional injections. The production of IgG is dependent on IgM stimulation and it is a secondary and more effective immune response to foreign materials in the body [41]. The IgG level was tested two weeks after the second injection and the second injection was 7 days after the first injection. As shown in Fig. 5B, DSPE-PEG groups induced the production of IgG, which was about 2.5 times of that for PBS group. In contrast, there was no significant difference between the PBS groups and the DSPE-PCB ${ }_{20}$ lipoplexes groups. The result further confirmed that the DSPE-PCB could avoid the stimulation of ABC phenomenon.

To further determine whether the generated IgM specifically bound to PEGylated lipoplexes, the PEGylated and PCBylated lipoplexes were incubated with the plasma obtained from rats received DSPE-PEG lipoplexes 5 days later. As shown in Figure 6 , the IgM level was significantly decreased after incubation with the DSPE-PEG lipoplexes, while there was no difference of IgM level after incubation with DSPE-PCB 20 lipoplexes. The results supported the idea that the elevated IgM selectively bound to DSPE-PEG lipoplexes and activated the ABC phenomenon. In contrast, PCBylation could avoid the $\mathrm{ABC}$ phenomenon and extend the blood circulation time of lipoplexes.
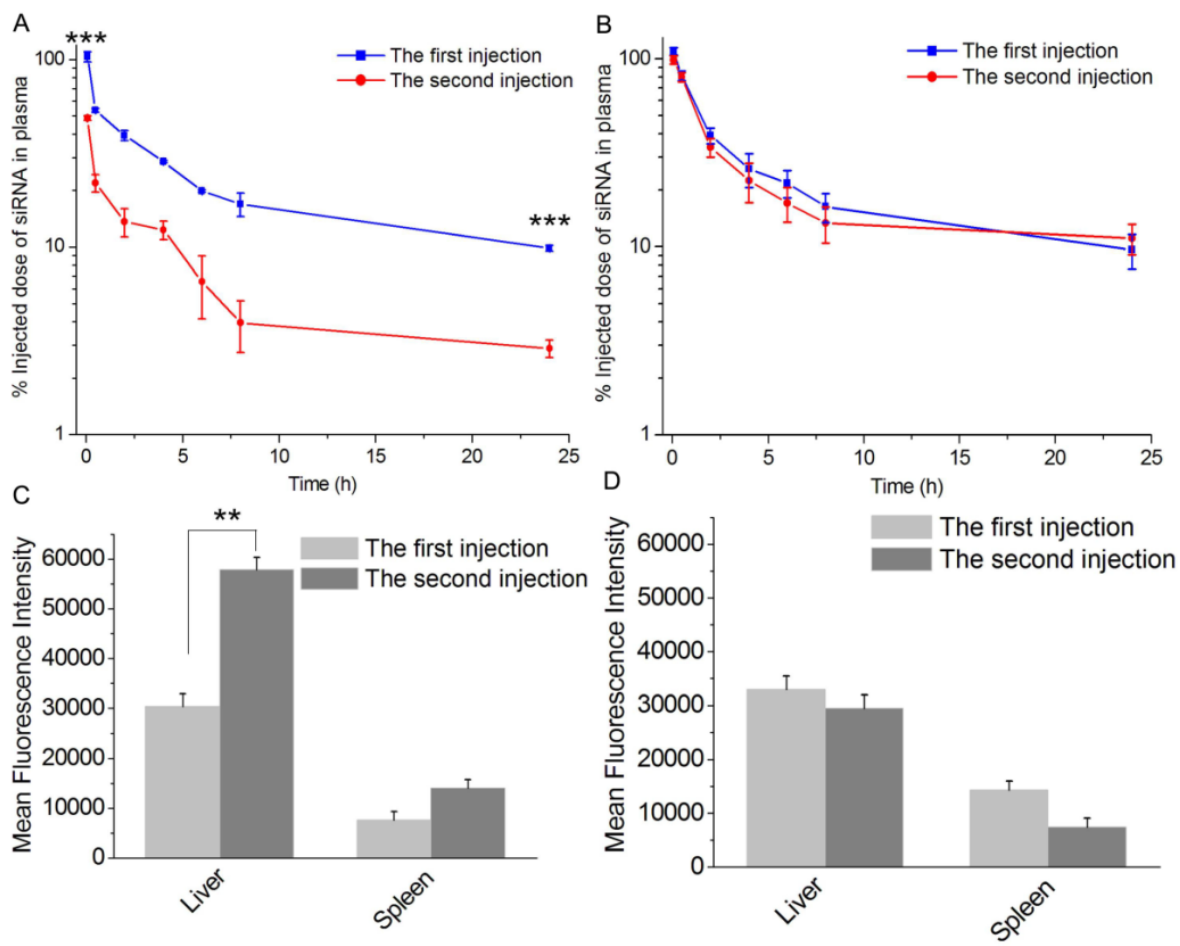

Figure 4. Pharmacokinetic and biodistribution study of DSPE-PEG and DSPE-PCB 20 lipoplexes in rats after two dose of injection with time interval of 5 days. (A) Blood clearance profile of DSPE-PEG lipoplexes. (B) Blood clearance profile of DSPE-PCB 20 lipoplexes. (C) Distribution of DSPE-PEG lipoplexes in liver and spleen. (D) Distribution of DSPE-PCB 20 lipoplexes in liver and spleen. Lipoplexes at N/P ratio of 5/1 were administered to SD rats with Cy5-labeled siRNA concentration of $1 \mathrm{mg} / \mathrm{kg}$ via the tail vein. Data are shown as the mean \pm S.D. of three independent experiments. $* P<0.05, * * P<0.01, * * * P<0.005(\mathrm{n}=3)$. 

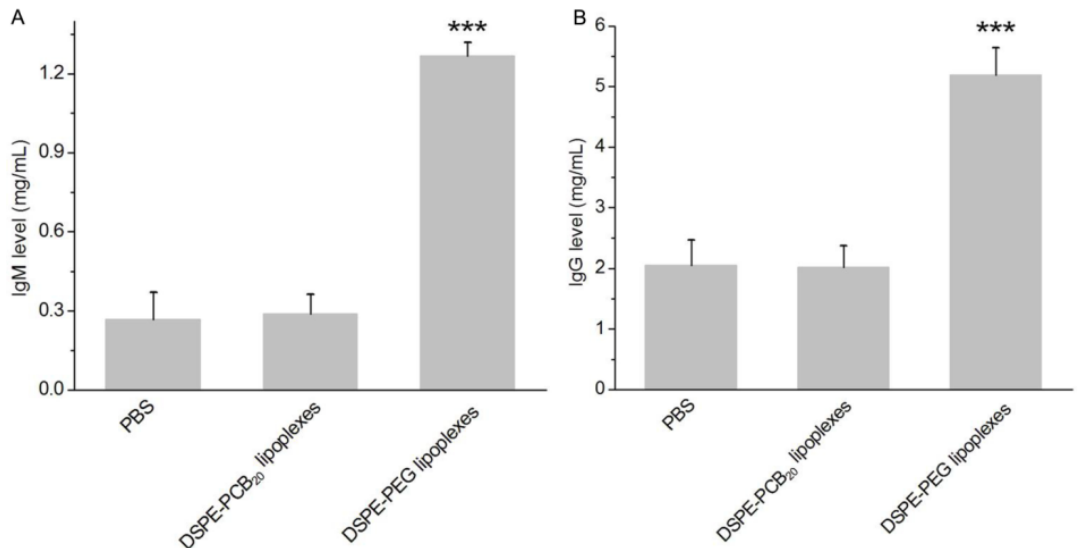

Figure 5. (A) IgM level in plasma of SD rats following administrated with DSPE-PEG and DSPE-PCB 20 lipoplexes 5 days later. (B) lgG level in plasma of SD rats following administrated with two injections of DSPE-PEG and DSPE-PCB 20 lipoplexes. The time interval between the two injections was 7 days and the plasma was obtained two weeks after the second injection. Lipoplexes at N/P ratio of 5/1 were administered to SD rats with Cy5-labeled siRNA concentration of $1 \mathrm{mg} / \mathrm{kg}$ via the tail vein. Data are shown as the mean \pm S.D. of three independent experiments. $* P<0.05, * * P<0.01, * * * P<0.005(n=5)$

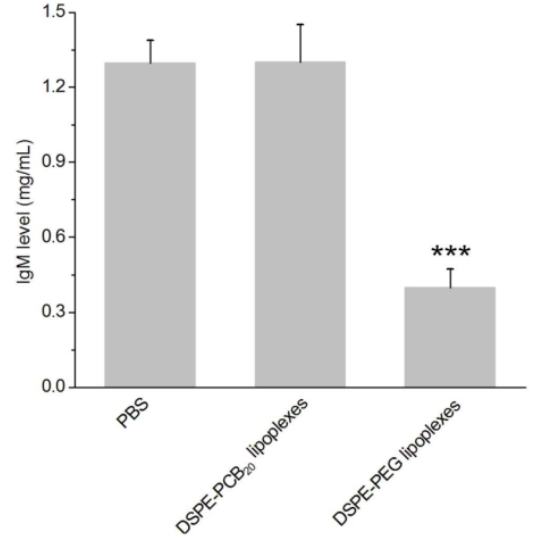

Figure 6. IgM level in plasma after incubation with DSPE-PEG and DSPE-PCB 20 lipoplexes at $37^{\circ} \mathrm{C}$ for $15 \mathrm{~min}$. The plasma was obtained from SD rats administrated with DSPE-PEG lipoplexes with Cy5-labeled siRNA concentration of 1 $\mathrm{mg} / \mathrm{kg}$. Data are shown as the mean \pm S.D. of three independent experiments. $* P<0.05, * * P<0.01, * * * P<0.005(n=3)$.

\section{Biodistribution of lipoplexes in tumor-bearing nude mice}

The $A B C$ phenomenon against the following dose of DSPE-PEG lipoplexes might interfere with its accumulation in tumor site. To demonstrate this, the biodistribution of lipoplexes with Cy5-labeled siRNA at $N / P$ ratio of $5 / 1$ was analyzed after two injections with a time interval of 5 days on Hela tumor-bearing nude mice. In comparison with naked Cy5-labeled siRNA, all lipoplexes formulations significantly enhanced the siRNA accumulation in tumor site due to the EPR effect. As shown in Figure 7A and 7C, the mean fluorescence intensity of Cy5-labeled siRNA in liver and tumor for DSPE-PEG and DSPE-PCB 20 was basic the same after the first injection. As expectedly, the mean fluorescence intensity of Cy5-labeled siRNA in liver for DSPE-PEG lipoplexes was about 1.5 times of that for DSPE-PCB 20 lipoplexes after the second injection, while the mean fluorescence intensity of Cy5-labeled siRNA in tumor for DSPE-PCB ${ }_{20}$ lipoplexes was about 1.3 times of that for DSPE-PEG lip- oplexes (Figure 7B and 7D). As shown in Figure 7E and $7 \mathrm{~F}$, the quantitative results further confirmed the results. The results demonstrated that DSPE- $\mathrm{PCB}_{20}$ avoided the $A B C$ phenomenon, which resulted in higher accumulation of Cy5-labeled siRNA in tumor for the following injection.

\section{Cellular uptake and intracellular distribution of lipoplexes}

After the lipoplexes accumulated in tumor site, the siRNA molecules must be transported into tumor cells, and more siRNA in the cytoplasm would induce more significant gene silencing effect [42]. The cellular uptake of DSPE-PCB 20 lipoplexes at N/P ratio of 5/1 was evaluated after incubation with Hela cells by flow cytometric analysis. As shown in Figure 8A, the fluorescence intensity of FAM-labeled siRNA in Hela cells was increased with the extension of culturing time from 0.25 to $2.5 \mathrm{~h}$. In addition, the fluorescence intensity of FAM-labeled siRNA for DSPE-PCB 20 lipoplexes was about 2 times of that for DSPE-PEG lipoplexes after $2.5 \mathrm{~h}$ incubation, indicating that DSPE-PCB ${ }_{20}$ lipoplexes had better cellular uptake ability than that of DSPE-PEG lipoplexes. This might be attributed to the unique chemical structure of DSPE-PCB ${ }_{20}$ that the cationic quaternary amine groups assisted the cationic liposomes in retaining the siRNA and cellular uptake.

To further probe the mechanisms underlying the cellular internalization of lipoplexes, the cellular uptake study was performed at lower temperature of 4 ${ }^{\circ} \mathrm{C}$ or in the presence of various endocytic inhibitors. As shown in Figure 8B, DSPE-PCB 20 and DSPE-PEG lipoplexes were internalized via energy dependent endocytosis, as cellular uptake of both lipoplexes was decreased about $70 \%$ at $4{ }^{\circ} \mathrm{C}$. In addition, chlorpromazine and $\mathrm{m} \beta \mathrm{CD}$ significantly inhibited the cellular uptake level of both lipoplexes, indicating that the lipoplexes were endocytosed via both the caveolaeand clathrin- mediated endocytosis pathways [43]. 


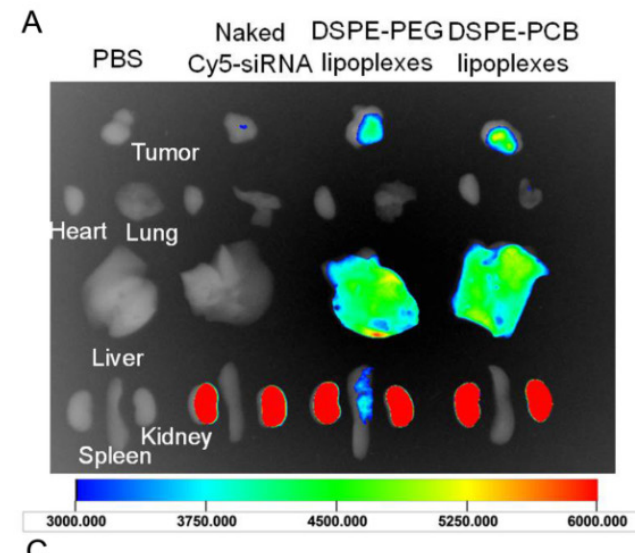

\section{B \\ PBS}

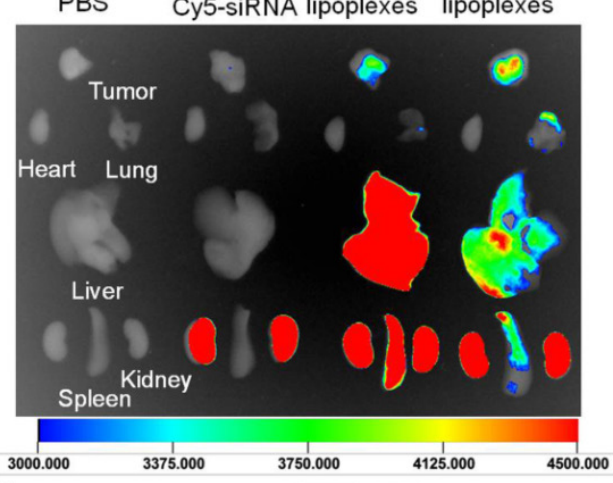

$\begin{array}{llll}3000.000 & 3375.000 & 3750.000 & 41250 \\ D^{* *} & \text { PBS }\end{array}$

6000 $\quad \prod^{* *}$ Naked siRNA
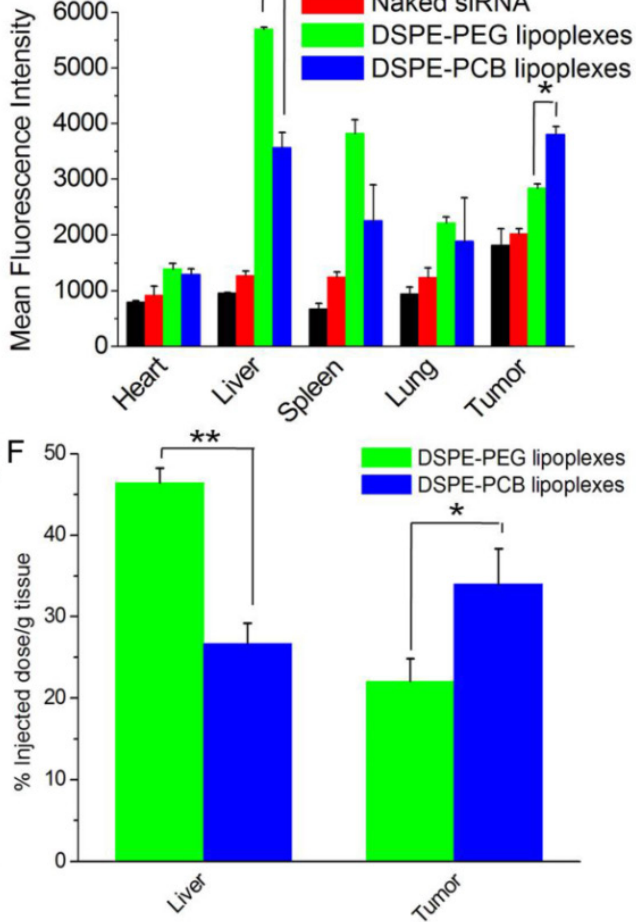

Figure 7. In vivo distribution of lipoplexes in Hela tumor-bearing nude mice after $24 \mathrm{~h}$ of two injections. (A) The fluorescence images of major organs and tumor after $24 \mathrm{~h}$ of the first injection. (B) The fluorescence images of major organs and tumor after $24 \mathrm{~h}$ of the second injection. (C) The mean fluorescence intensity of Cy5-labeled siRNA of major organs and tumor of Figure A. (D) The mean fluorescence intensity of Cy5-labeled siRNA of major organs and tumor of Figure B. (E) Quantitative results of Cy5-labeled siRNA in liver and tumor of the first injection. (F) Quantitative results of Cy5-labeled siRNA in liver and tumor of the second injection. Lipoplexes at N/P ratio of 5/l were administered to mice at a dose of $0.5 \mathrm{mg}$ Cy5-labeled siRNA/kg via the tail vein. Data are shown as the mean \pm S.D. of three independent experiments. $* P<0.05, * * P<0.01$, $* * * P<0.005(n=3)$.
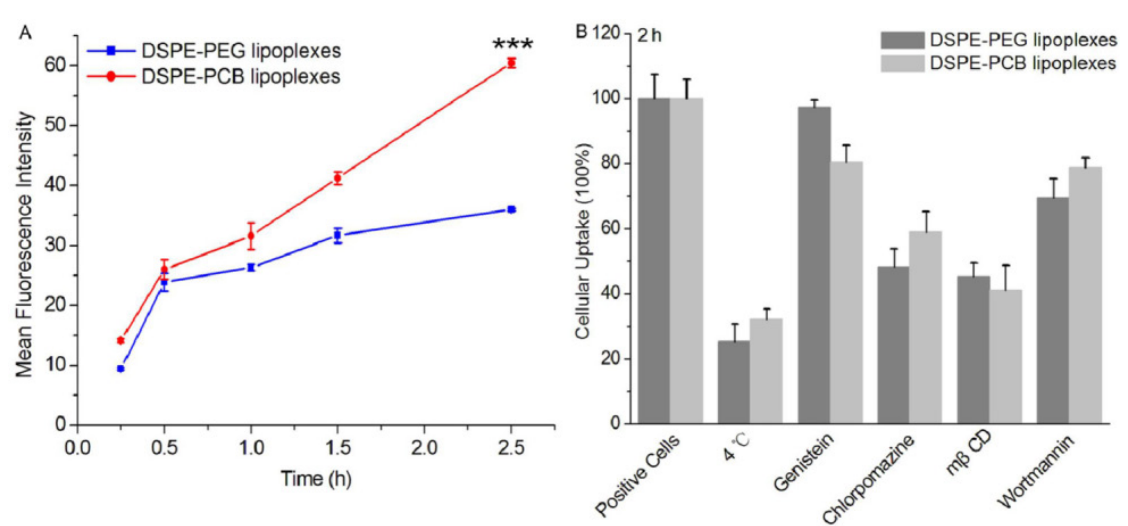

Figure 8. Flow cytometric analyses of cellular internalization of cationic liposome/FAM-labeled siRNA lipoplexes in Hela cells after incubation for different times. (A) Comparation of cellular internalization between DSPE-PEG and DSPE-PCB 20 lipoplexes with the extension of time. (B) Mechanistic probes of the intracellular kinetics of the DSPE-PEG and DSPE-PCB 20 lipoplexes by monitoring the cellular uptake level at $4{ }^{\circ} \mathrm{C}$ or in the presence of various endocytic inhibitors. Data are shown as the mean \pm S.D. of three independent experiments. 
As lipoplexes enter cells by an endocytosis pathway, the endosomal/lysosomal escape of siRNA is important for the subsequent post-transcriptional gene silencing in the cytoplasm [44]. To investigate whether siRNA loaded lipoplexes could efficiently escape from endosomes/lysosomes, Hela cells were treated with DSPE-PEG and DSPE-PCB 20 cationic liposome/FAM-labeled siRNA complexes at N/P ratio of $5 / 1$ for different periods of time, and the localization of lipoplexes in cells was observed by confocal laser scanning microscopy (CLSM). The endosomes/lysosomes were stained with LysoTracker Red, and the cell nuclei were counterstained with DAPI. As shown in Figure 9A, both DSPE-PEG and DSPE-PCB 20 lipoplexes were mainly colocalized with the LysoTracker Red stained organelles after $2 \mathrm{~h}$ of incubation, suggesting that lipoplexes resided in endosomes/lysosomes. However, after $8 \mathrm{~h}$ of incubation, the separation of the green and red fluorescence was more significant when the cells were incubated with DSPE-PCB ${ }_{20}$ lipoplexes, suggesting that FAM-labeled siRNA more efficiently escaped from the endosomes/lysosomes following the delivery of DSPE-PCB 20 lipoplexes. In contrast, the FAM-labeled
siRNA still resided in endosomes/lysosomes for DSPE-PEG lipoplexes after $8 \mathrm{~h}$ of incubation. The quantitative results of co-localization ratio for DSPE-PCB 20 lipoplexes decreased about $41.6 \%$, while only $22.8 \%$ for DSPE-PEG lipoplexes after $8 \mathrm{~h}$ incubation (Figure 9B). The percentage of released FAM-labeled siRNA from cationic liposomes of all internalized siRNA at various time points also confirmed the expedited endosomal/lysosomal escape of DSPE-PCB ${ }_{20}$ lipoplexes. As shown in Figure 9C, the cumulative release of siRNA at $8 \mathrm{~h}$ was $53.6 \pm 2.0 \%$ for $\mathrm{DSPE}^{-\mathrm{PCB}_{20}}$ lipoplexes, while only $12.9 \pm 1.4 \%$ for DSPE-PEG lipoplexes. As demonstrated in our previous work, the excellent endosomal/lysosomal escape ability of DSPE-PCB 20 lipoplexes was due to the $\mathrm{pH}$ sensitive ability of $\mathrm{PCB}_{20}$ that the carboxyl acid groups of $\mathrm{PCB}_{20}$ was protonation under acidic condition, resulting in increased zeta potential from $4.1 \pm 0.1$ $\mathrm{mV}$ at $\mathrm{pH} 7.4$ to $20.2 \pm 0.2 \mathrm{mV}$ at $\mathrm{pH} 3.5$ (Figure 9D). The increase in positive charge of DSPE- $\mathrm{PCB}_{20}$ lipoplexes promoted the fusion of lipoplexes with anionic endosomal/lysosomal membrane, and releasing siRNA into the cytoplasm [28].
A
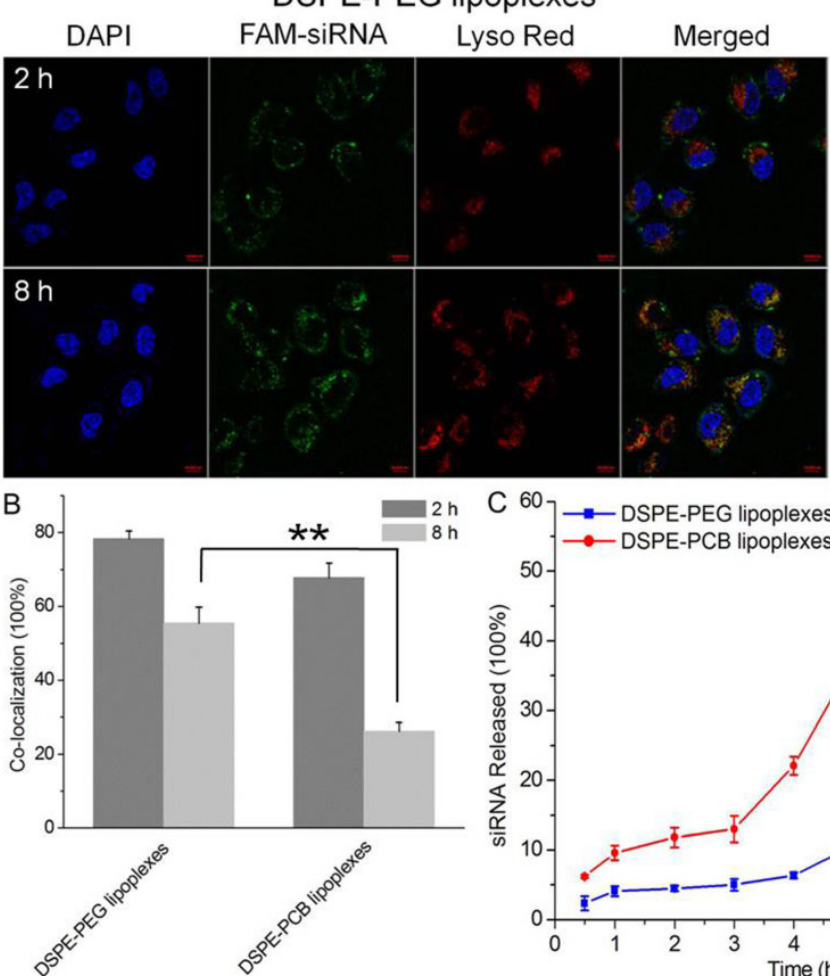

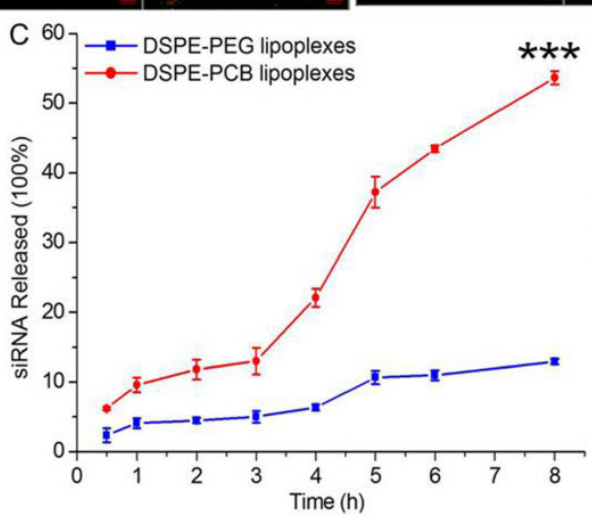

DSPE-PCB lipoplexes
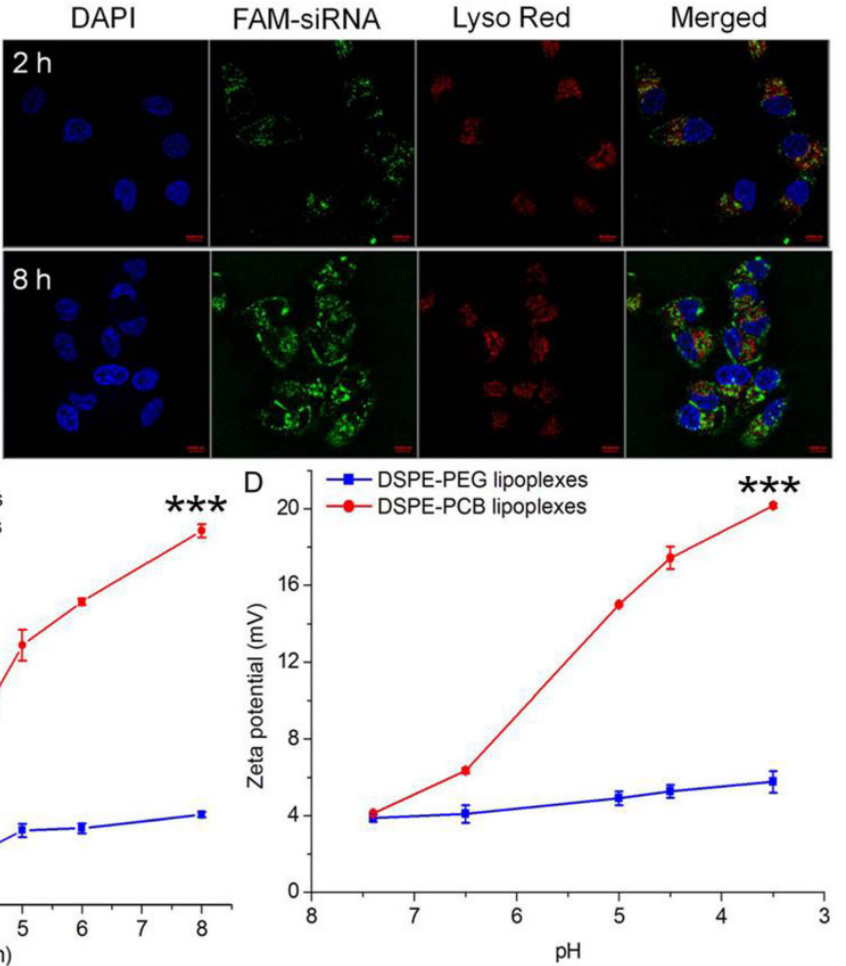

Figure 9. Assessment of cellular internalization and endosomal/lysosomal escape of lipoplexes in Hela cells after incubation for different time points. (A) Images detected by confocal laser scanning microscopy (CLSM). Cell nuclei and endosomes/lysosomes were stained with DAPI (blue) and LysoTracker Red (red). Scale bar is $10 \mu \mathrm{m}$. (B) Co-localization ratio of FAM-labeled siRNA and LysoTracker Red after $2 \mathrm{~h}$ and $8 \mathrm{~h}$ incubation. (C) The percentage of siRNA released from lipoplexes after incubation with the extension of time. (D) The changes of zeta potential of lipoplexes in different $\mathrm{PH}$ values PBs. Data are shown as the mean \pm S.D. of three independent experiments. 

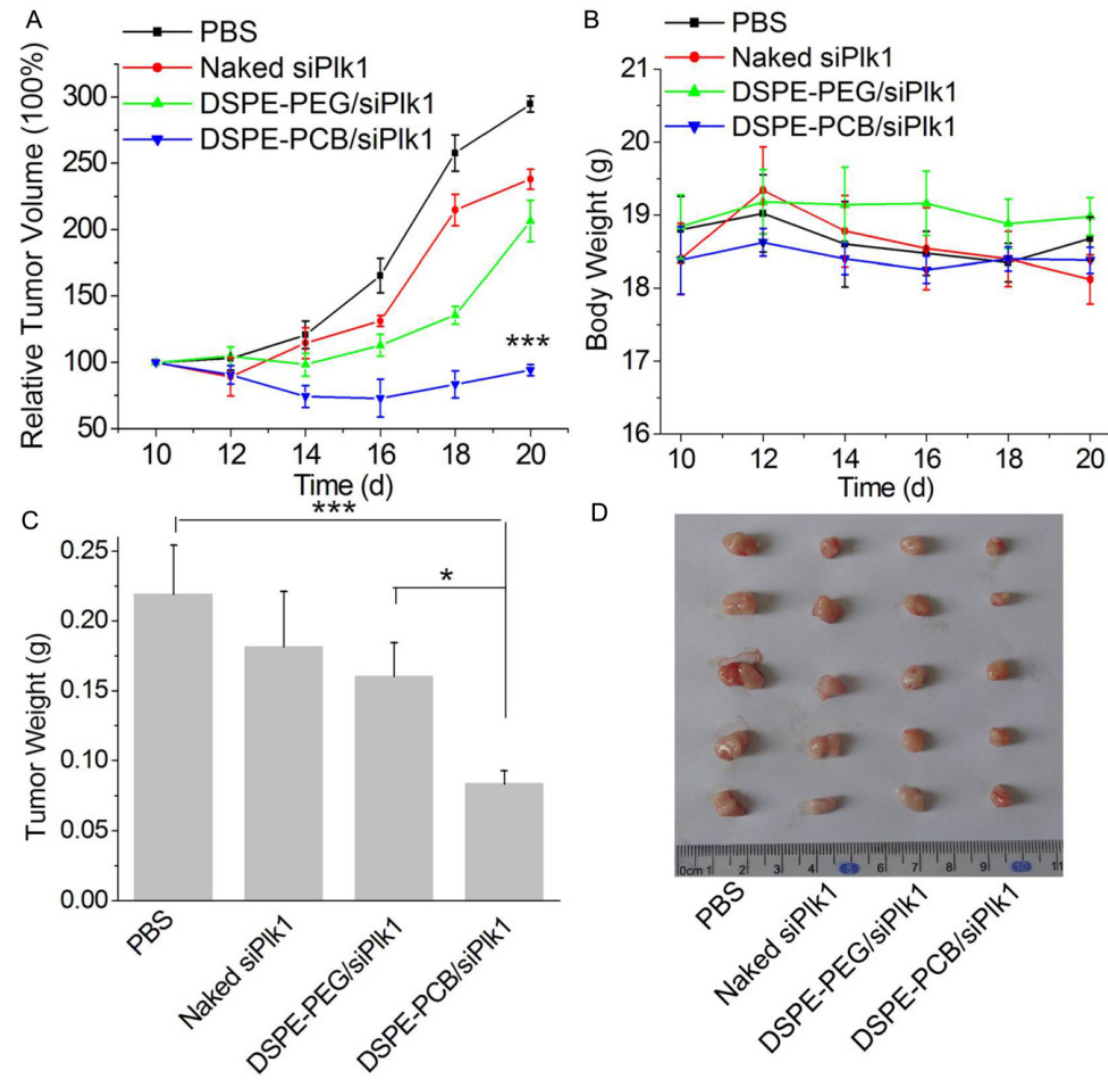

Figure 10. In vivo anti-tumor study of lipoplexes in Hela tumor-bearing nude mice after intravenous injection with PBS, siPIk1, DSPE-PEG and DSPE-PCB20 lipoplexes with siPlk1 at N/P ratio of $5 / 1$ at siPlkl dose of $2.5 \mathrm{mg} / \mathrm{kg}(\mathrm{n}=5)$. (A) Relative tumor volume-time curve. (B) Body weight-time curve. (C) The weights of the removed tumors. (D) The image of the solid tumors removed from different treatment groups. $* P<0.05, * * P<0.01, * * * P<0.005$.

\section{Tumor therapeutic activity of lipoplexes}

It has been demonstrated that PCBylation could avoid $\mathrm{ABC}$ phenomenon and enhance the tumor accumulation of lipoplexes after repeated injections in vivo. After the lipoplexes accumulating in tumor site, PCBylation could promote the siRNA molecules be transported into tumor cells, and accelerate its release into cytoplasm. To reveal the antitumor growth effect of DSPE-PCB 20 lipoplexes in vivo, Hela tumor-bearing nude mice were received intravenous injection of DSPE- $\mathrm{PCB}_{20}$ lipoplexes at siRNA dose of $2.5 \mathrm{mg} / \mathrm{kg}$. As illustrated in Figure 10A, treatment with siPlk1 alone did not show significantly tumor growth inhibition in comparison with PBS groups. The DSPE-PCB 20 lipoplexes with siPlk1 significantly inhibited tumor growth with the final relative tumor volume (RTV) of $94.2 \pm 4.2$, which was much lower than that of DSPE-PEG lipoplexes groups (206.5 \pm 15.6$)$. At the same time, both the DSPE-PEG and DSPE-PCB ${ }_{20}$ lipoplexes exhibited neglectable toxicity, which indicated the good biocompatibility of PEGylated and PCBylated lipoplexes (Figure 10B). The excised tumor weight also exhibited the corresponding weight (Figure 10C and 10D). The results indicated that DSPE-PCB 20 lipoplexes with siPlk1 exhibited excellent antitumor activity.
To demonstrate that retarded tumor growth by DSPE-PCB $_{20}$ lipoplexes was related to Plk1 down-regulation in tumor cells, the tumors were excised $24 \mathrm{~h}$ after the last injection. Tumor Plk1 mRNA and protein expression were analyzed by qRT-PCR and WB analyses, respectively. As shown in Figure $11 \mathrm{~A}$, treatment with siPlk1 alone did not show reductions in Plk1 mRNA level due to its lower tumor accumulation and cellular uptake. In contrast, Plk1 mRNA level showed a $80.4 \pm 2.5 \%$ reduction after treatment with DSPE-PCB 20 lipoplexes with siPlk1 compared to the level in tumors following treatment with PBS, which is much higher than that of DSPE-PEG lipoplexes (53.2 $\pm 3.8 \%)$. WB analyses of Plk1 protein levels in each tumor mass (Figure 11B and 11C) also revealed a significant reduction in Plk1 protein levels when the mice were treated with

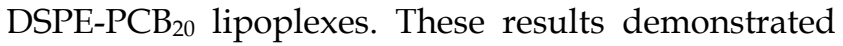
that DSPE-PCB ${ }_{20}$ lipoplexes could significantly down-regulate the Plk1 gene expression in vivo, which resulted in higher efficiency tumor growth suppression. Plk1 inhibition has been shown to be associated with apoptosis induction $[45,46]$. Cell apoptosis in the tumors after treatment with various formulations were analyzed by TUNEL (Figure 11D), that the DSPE-PCB ${ }_{20}$ lipoplexes with siPlk1 induced more significant cell apoptosis in tumors. 


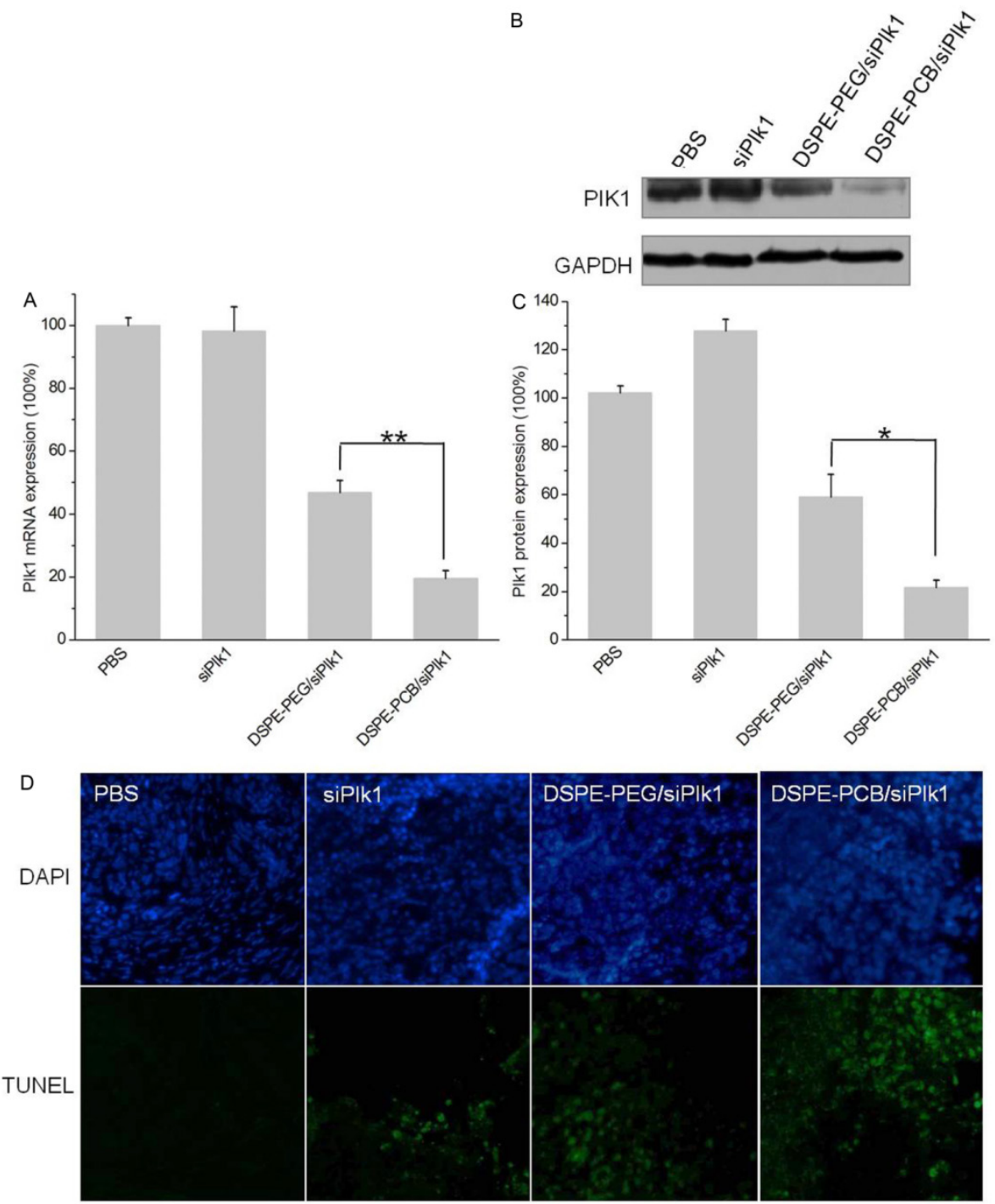

Figure 11. In vivo gene silencing efficiency of DSPE-PEG and DSPE-PCB 20 lipoplexes. (A) Expression of Plk1 mRNA. (B, C) Expression of Plk1 protein. (D) TUNEL analyses of tumor tissue after treatments. The tumor tissues were collected $24 \mathrm{~h}$ after the last injection. $* P<0.05, * * P<0.01, * * * P<0.005$.

\section{Conclusions}

We have developed zwitterionic polymer PCB modified cationic liposomes for the systemic delivery of siRNA therapeutics, which could overcome the barriers associated with the use of PEGylation. The PCBylated cationic liposomes could avoid ABC phenomenon, which extended the blood circulation time and enhanced the tumor accumulation of siRNA following injection. In addition, the DSPE- $\mathrm{PCB}_{20}$ cationic liposomes exhibited higher efficiency of siRNA encapsulation, cellular uptake and endosomal/lysosomal escape abilities, which in turn lead to remarkable release siRNA into cytoplasm. With excellent in vitro and in vivo behavior, the PCBylated cationic liposomes significantly inhibited tumor growth following systemic administration, suggesting its potential application for the delivery of siRNA therapeutics.

\section{Supplementary Material}

Figures S1 - S2.

http://www.thno.org/v05p0583s1.pdf

\section{Acknowledgments}

This work was financially supported by the National Natural Science Foundation of China (21304099, 51203162, 51103159, 51373177), the National High Technology Research and Development Program (2014AA020708, 2012AA022703, 2012AA020804), the Instrument Developing Project of the Chinese Academy of Sciences (YZ201253, YZ201313), the Open Funding Project of the National Key Laboratory of Biochemical Engineering (Y22504A169) and the "Strategic Priority Research Program" of the Chinese Academy of Sciences (XDA09030301-3). 


\section{Competing Interests}

The authors have declared that no competing interest exists.

\section{References}

1. Li Y, Liu RY, Yang J, et al. Dual sensitive and temporally controlled camptothecin prodrug liposomes codelivery of siRNA for high efficiency tumor therapy. Biomaterials. 2014; 35: 9731-45.

2. Takemoto $\mathrm{H}$, Miyata $\mathrm{K}$, Hattori $\mathrm{S}$, et al. Acidic $\mathrm{pH}$-responsive siRNA conjugate for reversible carrier stability and accelerated endosomal escape with reduced IFNa-associated immune response. Angew Chem Int Ed. 2013; 125: 6338-41

3. Yang CX, Nilsson L, Cheema MU, et al. Chitosan/siRNA nanoparticles targeting cyclooxygenase type 2 attenuate unilateral ureteral obstruction-induced kidney injury in mice. Theranostics. 2015; 5: 110-23.

4. Hong BJ, Chipre AJ, Nguyen ST. Acid-degradable polymer-caged lipoplex (PCL) platform for siRNA delivery: facile cellular triggered release of siRNA. J Am Chem Soc. 2013; 135: 17655-8.

5. Draz MS, Fang BA, Zhang PF, et al. Nanoparticle-mediated systemic delivery of siRNA for treatment of cancers and viral infections. Theranostics. 2014; 4: 872-92.

6. Liu HM, Li Y, Mozhi AB, et al. SiRNA-phospholipid conjugates for gene and drug delivery in cancer treatment. Biomaterials. 2014; 35: 6519-33.

7. Shen JL, Kim HC, Su H, et al. Cyclodextrin and polyethylenimine functionalized mesoporous silica nanoparticles for delivery of siRNA cancer therapeutics. Theranostics. 2014; 4: 487-97.

8. Yang T, Li B, Qi SB, et al. Co-delivery of doxorubicin and Bmil siRNA by folate receptor targeted liposomes exhibits enhanced anti-tumor effects in vitro and in vivo. Theranostics. 2014; 4: 1096-111.

9. Canine BF, Wang YH, Ouyang WY, et al. Development of targeted recombinant polymers that can deliver siRNA to the cytoplasm and plasmid DNA to the cell nucleus. J Control Release. 2011; 151: 95-101.

10. Simões S, Moreira IN, Fonseca $\mathrm{C}$, et al. On the formulation of $\mathrm{pH}$-sensitive liposomes with long circulation times. Adv Drug Deliv Rev. 2004; 56: 947-65.

11. Tseng YC, Mozumdar S, Huang L. Lipid-based systemic delivery of siRNA. Adv Drug Deliv Rev. 2009; 61: 721-31.

12. Kenny GD, Kamaly N, Kalber TL, et al. Novel multifunctional nanoparticle mediates siRNA tumour delivery, visualisation and therapeutic tumour reduction in vivo. J Control Release. 2011; 149: 111-6.

13. Sakurai Y, Hatakeyama H, Sato $Y$, et al. Endosomal escape and the knockdown efficiency of liposomal-siRNA by the fusogenic peptide shGALA. Biomaterials. 2011; 32: 5733-42.

14. Hong CA, Nam YS. Functional nanostructures for effective delivery of small interfering RNA therapeutics. Theranostics. 2014; 4: 1211-32.

15. Chan CL, Majzoub RN, Shirazi RS, et al. Endosomal escape and transfection efficiency of PEGylated cationic liposome-DNA complexes prepared with an acid-labile PEG-lipid. Biomaterials. 2012; 33: 4928-35.

16. Ishida $T$, Maeda $R$, Ichihara $M$, et al. Accelerated clearance of PEGylated liposomes in rats after repeated injections. J Control Release. 2003; 88: 35-42.

17. Tagami T, Nakamura K, Shimizu $T$, et al. Effect of siRNA in PEG-coated siRNA-lipoplex on anti-PEG IgM production. J Control Release. 2009; 137: 234-40.

18. Zhao YX, Wang L, Yan MN, et al. Repeated injection of PEGylated solid lipid nanoparticles induces accelerated blood clearance in mice and beagles. Int J Nanomedicine. 2012; 7: 2891-900.

19. Tagami T, Uehara Y, Moriyoshi N, et al. Anti-PEG IgM production by siRNA encapsulated in a PEGylated lipid nanocarrier is dependent on the sequence of the siRNA. J Control Release. 2011; 151: 149-54

20. Ishida T, Harada M, Wang XY, et al. Accelerated blood clearance of PEGylated liposomes following preceding liposome injection: effects of lipid dose and PEG surface-density and chain length of the first-dose liposomes. J Control Release. 2005; 105: 305-17.

21. Koide $\mathrm{H}$, Asai T, Hatanaka $\mathrm{K}$, et al. T cell-independent $\mathrm{B}$ cell response is responsible for $\mathrm{ABC}$ phenomenon induced by repeated injection of PEGylated liposomes. Int J Pharm. 2010; 392: 218-23.

22. Ishihara T, Maeda T, Sakamoto $\mathrm{H}$, et al. Evasion of the accelerated blood clearance phenomenon by coating of nanoparticles with various hydrophilic polymers. Biomacromolecules. 2010; 11: 2700-6.

23. Lila ASA, Kiwada H, Ishida T. The accelerated blood clearance (ABC) phenomenon: clinical challenge and approaches to manage. J Control Release 2013; 172: 38-47.

24. Semple SC, Harasym TO, Clow KA, et al. Immunogenicity and rapid blood clearance of liposomes containing polyethylene glycol-lipid conjugates and nucleic acid. J Pharmacol Exp Ther. 2005; 312: 1020-6.

25. Xu H, Wang KQ, Deng YH, et al. Effects of cleavable PEG-cholesterol derivatives on the accelerated blood clearance of PEGylated liposomes. J Control Release. 2010; 31: 4757-63.

26. Zhai XY, Wang W, Wang CD, et al. PDMAEMA-b-polysulfobetaine brushes-modified $\varepsilon$-polylysine as a serum-resistant vector for highly efficient gene delivery. J Mater Chem. 2012; 22: 23576-86.
27. Cheng L, Li YM, Zhai XY, et al. Polycation-b-polyzwitterion copolymer grafted luminescent carbon dots as a multifunctional platform for serum-resistant gene delivery and bioimaging. ACS Appl Mater Inter. 2014; 6: 20487-97.

28. Li Y, Cheng Q, Jiang Q, et al. Enhanced endosomal/lysosomal escape by distearoyl phosphoethanolamine-polycarboxybetaine lipid for systemic delivery of siRNA. J Control Release. 2014; 176: 104-14.

29. Li SD, Chen YC, Hackett MJ, et al. Tumor-targeted delivery of siRNA by self-assembled nanoparticles. Mol Ther. 2008; 16: 163-9.

30. Dai FY, Liu WG. Enhanced gene transfection and serum stability of polyplexes by PDMAEMA-polysulfobetaine diblock copolymers. Biomaterials. 2011; 32: 628-38.

31. Hatakeyama $\mathrm{H}$, Akita $\mathrm{H}, \mathrm{Harashima} \mathrm{H}$. A multifunctional envelope type nano device (MEND) for gene delivery to tomours based on the EPR effect: a strategy for overcoming the PEG dilemma. Adv Drug Deliv Rev. 2011; 63: 152-60.

32. Huang SX, Shao K, Liu Y, et al. Tumor-targeting and microenvironment-responsive smart nanoparticles for combination therapy of antiangiogenesis and apoptosis. ACS Nano. 2013; 7: 2860-71.

33. Lee $\mathrm{CH}, \mathrm{Ni} \mathrm{YH}, \mathrm{Chen} \mathrm{CC}$, et al. Synergistic effect of polyethylenimine and cationic liposomes in nucleic acid delivery to human cancer cell. BBB-Biomembranes. 2003; 1611: 55-62.

34. Dauty E, Remy JS, Blessing T, et al. Dimerizable cationic detergents with a low cmc condense plasmid DNA into nanometric particles and transfect cells in culture. J Am Chem Soc. 2001; 123: 9227-34.

35. Meyer O, Kirpotin D, Hong K, et al. Cationic liposomes coated with polyethylene glycol as carriers for oligonucleotides. J Biol Chem. 1998; 273: 15621-27.

36. Laverman P, Carstens MG, Boerman OC, et al. Factors affecting the accelerated blood clearance of polyethylene glycol-liposomes upon repeated injection. J Pharmacol Exp Ther. 2001; 298: 607-12

37. Ishihara $\mathrm{T}$, Takeda $\mathrm{M}$, Sakamoto $\mathrm{H}$, et al. Accelerated blood clearance phenomenon upon repeated injection of PEG-modified PLA-nanoparticles. Pharm Res. 2009; 26: 2270-9.

38. Ishida $\mathrm{T}$, Ichihara $\mathrm{M}$, Wang $\mathrm{XY}$, et al. Spleen plays an important role in the induction of accelerated blood clearance of PEGylated liposomes. J Control Release. 2006; 115: 243-50.

39. Ishida T, Atobe K, Wang XY, et al. Accelaerated blood clearance of PEGylated liposomes upon repeated injections: effect of doxorubicin-encapsulation and high-dose first injection. J Control Release. 2006; 115: 251-8.

40. Lila ASA, Nawata K, Shimizu T, et al. Use of polyglycerol (PG), instead of polyethylene glycol (PEG), prevents induction of the accelerated blood clearance phenomenon against long-circulating liposomes upon repeated administration. Int J Pharm. 2013; 456: 235-42.

41. Sroda K, Rydlewski J, Langner M, et al. Repeated injections of PEG-PE liposomes generate anti-PEG antibodies. Cell Mol Biol Lett. 2005; 10: 37-47.

42. Yang XZ, Dou S, Sun TM, et al. Systemic delivery of siRNA with cationic lipid assisted PEG-PLA nanoparticles for cancer therapy. J Control Release. 2011; 156: 203-11.

43. Yin LC, Song ZY, Kim KH, et al. Reconfiguring the architectures of cationic helical polypeptides to control non-viral gene delivery. Biomaterials. 2013; 34: 2340-9.

44. Obata Y, Tajima S, Takeoka S. Evaluation of $\mathrm{pH}$-responsive liposomes conaining amino acid-based zwitterionic lipids for improving intracellular drug delivery in vitro and in vivo. J Control Release. 2010; 142: 267-76.

45. Yang XZ, Dou S, Wang YC, et al. Single-step assembly of cationic lipid-polymer hybrid nanoparticles for systemic delivery of siRNA. ACS Nano. 2012; 6: 4955-65.

46. Yang XZ, Du JZ, Dou S, et al. Sheddable ternary nanoparticles for tumor acidity-targeted siRNA delivery. ACS Nano. 2012; 6: 771-81. 